\title{
Maintenance of the Photovolatic Plants Using UAV Equipped with Low-cost GNSS RTK Receiver
}

\author{
Bilal Muhammad ${ }^{1}$, Ramjee Prasad ${ }^{1}$, Marco Nisi $^{2}$, Fabio Menichetti ${ }^{2}$, \\ Ernestina Cianca ${ }^{3}$, Alberto Mennella ${ }^{4}$, Graziano Gagliarde ${ }^{4}$ \\ and Davide Marenchino ${ }^{5}$ \\ ${ }^{1}$ Department of Business Development and Technology, \\ Aarhus University, Denmark \\ ${ }^{2}$ Sistematica S.p.a, Italy \\ ${ }^{3}$ CTIF Section, Department of Electronics, \\ University of Rome Tor Vergata, Italy \\ ${ }^{4}$ TopView srl, Italy \\ ${ }^{5}$ Entec S.p.a, Italy
}

Received 10 February 2017; Accepted 31 May 2017; Publication 19 February 2018

\begin{abstract}
Global Navigation Satellite System (GNSS) Real Time Kinematic (RTK) employs high-end dual-frequency receivers and antennas to deliver precise positioning that, in some way, restricts the use of GNSS RTK to a subset of user market due to very high cost. The emerging mass-market user applications, however, require centimeter-positioning accuracy considering a cost-effective solution. This calls for low-cost GNSS RTK solutions to create new possibilities for mass-market user applications to make use of GNSS high accuracy positioning in a variety of ways. One of the applications, which makes use of low-cost GNSS RTK receiver, is the maintenance of photovoltaic (PV) plants using Unmanned Aerial Vehicle (UAV). This paper proposes a solution that aims at automating the maintenance of PV plant with enhanced reliability in a time and cost effective manner, which otherwise requires intermediate human intervention. The paper presents the architectural concept, system design, and
\end{abstract}

Journal of Communication, Navigation, Sensing and Services, Vol. 1, 25-48. doi: 10.13052/jconasense2246-2120.2017.002

This is an Open Access publication. (c) 2018 the Author(s). All rights reserved. 
end-to-end algorithm that plays a pivotal role in enabling the automatic report generation of PV plant status. Preliminary results of the proof-of-concept shows the feasibility of the proposed solution.

Keywords: Photovoltaic, UAV, GNSS, RTK.

\section{Introduction}

PV plant owners are responsive about enhancing their energy production and recognize the importance of maintenance in the life cycle of a PV plant according to the policies of incentives activated in the past years inside the European Union member states in favor of clean energy production. PV plants experience aging over a period of time that results in lower production mainly due to the inefficiencies caused by faults or defects in unknown modules. One way of inspection of faulty modules in a PV plant is performed using handheld thermal camera, kept by a person walking over the whole PV plant, on the ground or on the rooftop. This method has itself significant drawbacks, for instance, the need for ground plants, walking over large spaces of wild grass, snow, and rough terrain. Another maintenance approach is realized by the installation of expensive system, which consists of various sensors communicating with a center station. Such a method allows the detection of faulty PV panel but does not provide information of the unique faulty module within the detection PV panel. Therefore, human intervention would eventually be needed to locate the faulty module within the identified PV panel. The maintenance becomes even more cumbersome for those PV plants that are installed in remote areas where communication and surveillance infrastructure is hard to deploy; consequently a dedicated crew or service is required that contributes to the overall maintenance cost.

To minimize the operational complexities in maintaining a PV plant, the proposed solution presented in this paper aims at automating the overall process of maintenance by employing a UAV equipped with a thermal camera and low-cost GNSS RTK receiver, which performs a mission flying over a photovoltaic field and collecting optical and thermal images. The collected data processed by means of a computer vision algorithm and referenced by means of on-board L1 GNSS RTK receiver would allow the maintainer to identify and locate the defective module to be replaced without intermediate human inspections.

The organization of the paper is as follows. Section 2 describes the proposed solution system architecture concept, system design, end-to-end 
system algorithm, and subsystem error sources. Section 3 focuses on GNSS centimeter positioning accuracy requirement and potential solutions to meet the positioning accuracy requirement. Section 4 presents preliminary results and analysis that have been carried out so far, followed by conclusion in Section 5 .

\section{Proposed Solution}

The proposed solution aims at automating the maintenance of PV plant with enhanced reliability in a time and cost effective manner from an operational perspective. This purpose shall be achieved by enhancing the overall safety during the on-field inspection process using a custom UAV payload design for PV plants maintenance to avoid the employment of people checking modules on rooftop plants (running risks for their safety) or in ground plants (sometimes crossing rough terrain and tall grass).

\subsection{System Architecture}

A depiction of the system architecture is presented in Figure 1. As shown, the proposed solution employs UAV equipped with a thermal camera, low-cost GNSS RTK receives, and visual sensor. The UAV shall perform a mission flying over a photovoltaic field to collect optical and thermal images of solar

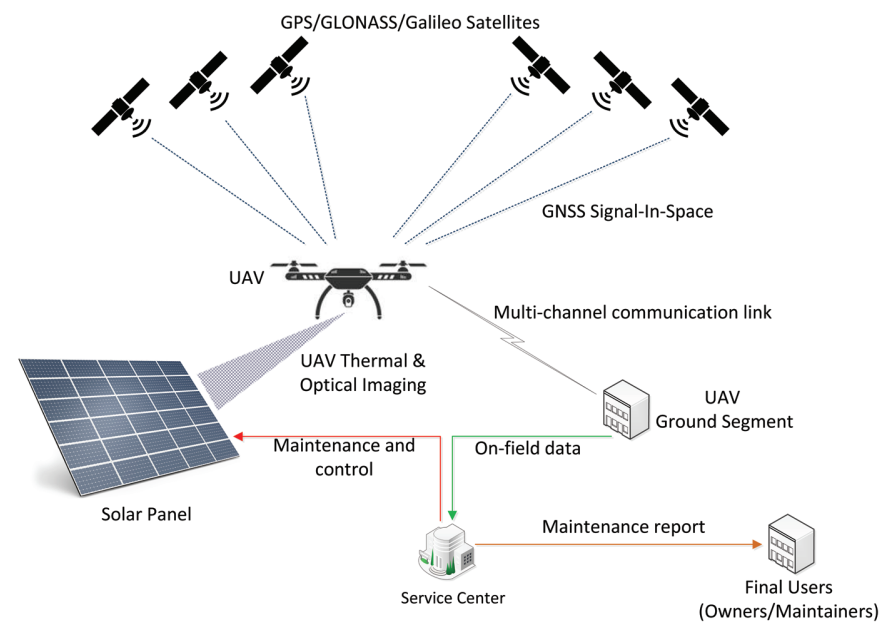

Figure 1 System architecture concept of the proposed solution. 
panels in a PV plant. The data collected data by UAV shall be processed by means of a computer vision algorithm, and the captured images shall be tagged by means of centimeter-level position fix provided by L1 RTK GNSS receiver. The centimeter-level positioning provided by GNSS enables the automation of the entire process allowing to correctly geo-reference the defective panel inspected by the on-board thermal camera. Finally, this information shall be provided to the remote service center in charge of thermal anomalies identification and management.

\subsection{Subsystem Design}

The proposed system is composed of three subsystems:

1. Service center

2. UAV

3. UAV Ground Segment

\subsubsection{Service center}

Service center is devoted to manage all aspects related to production, delivery, archiving and cataloguing of data acquired and processed. It performs:

- Product Archiving and Cataloguing of mission data such as Optical Images, Thermal Images, Videos and post processed products. It includes retrieval of previously archived and cataloged data/products and catalogue browsing.

- Mission Planning to arrange organization and cooperation between the various entities involved throughout the mission process.

- Post Processing Management of acquired/catalogued products. These activities can be operated in automatic mode or manually by Thermographic Expert Operator (TEO).

- Product Validation before delivery is a function mainly used by the TEO to see the products, processing and making analysis, and write annotations, etc.

- Product Delivery and Distribution to the final user of validated products, including report formatting, archiving and distribution.

- Monitoring and Control including all the activities involved in the management and presentation of information coming from the PV plant such as monitoring and control of plant elements status, alarm supervision and production parameters supervision. 


\subsubsection{UAV}

A block diagram of UAV subsystem is provided in Figure 2. As shown, UAV is composed of COTS (Commercially Of the Shelf) or self-built drone platform, Communication and Control $(\mathrm{C} \& \mathrm{C})$ unit, and the UAV Payload. The UAV Payload is consist of the following units:

- On-Board Computer (OBC) responsible for interfacing and handling the payload sensors (Barometer, GNSS RTK Receiver, IMU, Thermal and Optical Camera), storing thermal and visual video/image, georeferecning, and enriching each frame with ancillary information such as GNSS positioning fix status, attitude and related metadata.

- ublox M8P L1 RTK GNSS receiver equipped with Tallysman TW3400 patch antenna, which acts as a rover. M8P supports dual-constellation GNSS operation at a position update rate of up to $5 \mathrm{~Hz}$.

- 2 or 3 axis mechanical gimbal with its dedicated electronic control unit, which is designed for housing thermal sensor and optionally visual sensors with a custom design.

- Thermal and visual sensors implemented by commercial sensors (COTS) available in the market, such as, FLIR VUE PRO or FLIR TAU2.

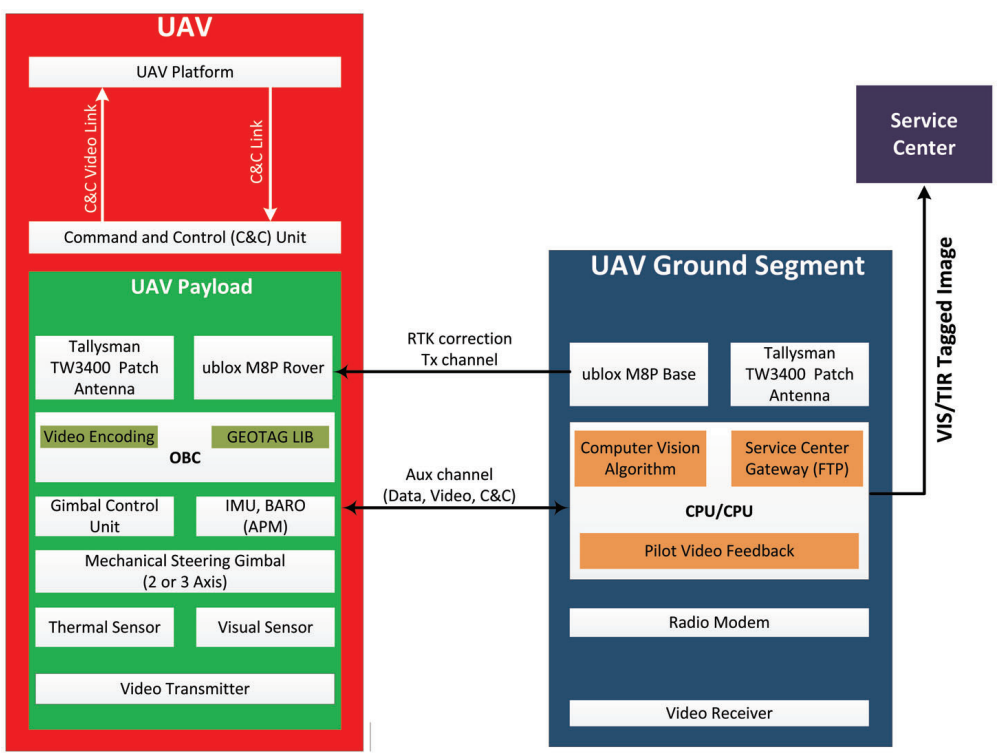

Figure 2 UAV and UAV ground subsystem design. 


\subsubsection{UAV ground segment}

The UAV ground segment is composed of the following:

- Central Processing Unit (CPU) with dedicated Graphical Processing Unit (GPU) to perform computationally intensive image processing. As a first choice, Jetson Tx1 (256GPU Cuda cores) development board based on Nvidia Tegra processor has been selected. The software modules within CPU are:

- Computer vision algorithm performs various operations (offline) on the data collected by UAV, which include detection of thermal anomalies and identification of solar panels with unique ID based on its geographic placement using precise GNSS position fix and related metadata. The computer vision algorithm can be implemented over a distributed architecture by porting some of the recognition capabilities on-board UAV.

- Pilot Video Feedback acts as a real-time video for the UAV pilot, which enables the pilot to have a real-time feedback of panels irradiation and an estimation of the area covered directly during on-field operations.

- Service Center gateway is responsible for the generation of all the application and transport layers towards service center, according to the defined protocol (e.g. FTP/XML).

- GNSS receiver module at UAV ground segment is ublox M8P L1 RTK receiver equipped with Tallysman TW3400 patch antenna, which acts as a RTK base station providing RTK correction in RTCM (v.3.x) format to the UAV through a UHF radio link or internet modem.

- Radio Modem is used for receiving the real time ancillary data (AUX channel - NAVCOM data, attitude, etc.) that will be used by the pilot for various operational purposes.

\subsection{End-to-End Algorithm}

The end-to-end algorithm combines centimeter-level RTK GNSS receiver positioning capabilities with computer vision and geodesics algorithms largely used in the literature [1-4] to identify and locate the faulty solar panel modules. The algorithm performs the analysis of acquired PV panels images to detect possible thermal anomalies and subsequently uses direct geo-referencing of images acquired through infrared (IR) camera to identify the geographical location of defective modules. Finally, a database (DB) is created by assigning 
each panel a unique identifier based on its precise geographical placement. The key steps performed during the process are summarized as below.

\subsubsection{Image acquisition}

The image acquisition shall be performed by implementing nadiral acquisition photogrammetric technique, as described in $[5,6]$. The image is acquired by IR camera when the optical axis of camera is perpendicular to the plane formed by $\mathrm{X}_{o b j}$ and $\mathrm{Y}_{o b j}$. The plane formed by $\mathrm{X}_{o b j}$ and $\mathrm{Y}_{o b j}$ is referred to as Eulerian object reference system, which a right oriented East, North, Up (ENU) originated in an arbitrary known point $O\left(x_{0}, y_{0}, z_{0}\right)$ as illustrated in Figure 3. In order to keep the optical axis of the camera normal to the object space, on-board gimbal adjustment shall be performed using the information obtained from on-board sensors.

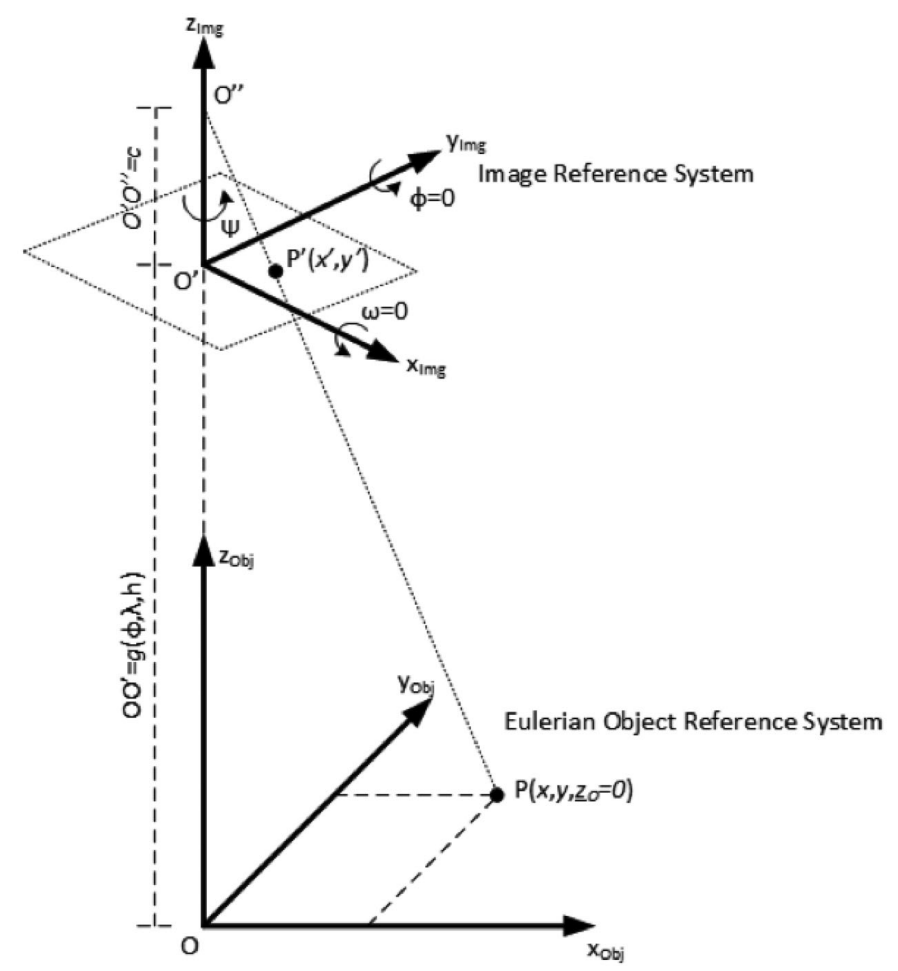

Figure 3 Geometric illustration of Eulerian object reference system and image reference system. 


\subsubsection{Direct geo-referencing}

In order to geo-reference every point $\mathrm{P}_{\left(x, y, z_{0=0}\right)}$ acquired with the IR camera in the object space direct geo-referencing image algorithms shall be implemented [7]. Considering that the object is placed in the planer surface (2-dimensional) and the optical axis of the camera is perpendicular to the object space, the geo-referencing of point $\mathrm{P}_{\left(x, y, z_{0}=0\right.}$ is the same as knowing $(x, y)$ scalar parameters of the image. For this purpose, a reference system, which is referred to as the image reference system is defined as shown in Figure 3. The image reference system is a right oriented reference system originated in $O^{\prime}$ while having $\mathrm{Z}_{i m g}$ axis parallel to $\mathrm{Z}_{o b j}$ and $\mathrm{X}_{i m g}$ and $\mathrm{Y}_{i m g}$ forms a plane parallel to $\mathbf{X}_{o b j}$ and $\mathrm{Y}_{o b j}$ axis. Furthermore $\mathbf{X}_{i m g} \cdot \mathbf{X}_{o b j}=\cos (\Psi)$ and $\mathrm{P}_{\left(x, y, z_{0}=0\right)}$ becomes $\mathrm{P}_{\left(x^{\prime} y^{\prime}\right)}^{\prime}$ image space. The aforementioned process of geo-referencing is performed by a transformation function $\mathscr{F}(\cdot)$ given in Equation (1).

$$
\mathrm{P}_{\left(\mathrm{x}, \mathrm{y}, \mathrm{z}_{0}=0\right)}^{\mathrm{k}}=\mathscr{F}\left(\mathrm{P}_{\left(\mathrm{x}^{\prime} \mathrm{y}^{\prime}\right)}^{\prime \mathrm{k}}, \mathrm{c}, \Psi, \mathrm{h}, \phi_{\mathrm{c}}, \lambda_{\mathrm{c}}\right)
$$

Where:

- $\mathrm{P}_{\left(x, y, z_{0=0}\right)}^{k}$ is the point in object space.

- $\mathrm{P}_{\left(x^{\prime} y^{\prime}\right)}^{\prime}$ is point (in pixel) in the image space provided by the camera sensor.

- $\mathrm{c}$ is the focal distance that depends upon the camera sensor.

- $\mathrm{h}$ is the UAV altitude obtained from on-board barometric sensor.

- $\phi_{c}$ is the longitude and $\lambda_{c}$ is the latitude of on-board moving camera sensor.

It has to be remarked that $\phi_{c}$ and $\lambda_{c}$ are obtained through a transformation function from on-board GNSS Antenna Phase Center (APC) latitude $\phi_{G N S S}$ and longitude $\lambda_{G N S S}$, which are accurate to centimeter level mainly due to RTK based positioning. This indicates the significance of centimeter level positioning in enabling the automation of PV plant maintenance.

Eventually, the point $\mathrm{P}_{\left(x, y, z_{0}=0\right)}^{k}$ in the object space shall be transformed from object reference system (ENU) to World Geodetic System (WGS84) representation of geographical coordinate as:

$$
\mathrm{P}_{(\phi, \lambda)}^{\mathrm{k}}=\mathscr{G}\left(\mathrm{P}_{\left(\mathrm{x}, \mathrm{y}, \mathrm{z}_{0}=0\right.}^{\mathrm{k}}\right)
$$

\subsubsection{Panel recognition and faulty objects detection}

The process of panels recognition and its related faulty objects (e.g. hotspot, hot strips and other kind of thermal anomalies) is implemented with image processing [8]. For our application, given a video sequence containing one or 
more faulty moving objects, the desired result is the set of the trajectories of these objects and an estimation of their position.

\subsubsection{Database cataloguing}

The PV Panels can be uniquely catalogued by the generation of an alphanumeric primary key exploiting the position information. Each panel, surrounded by its bounding box, is tracked by the algorithm $\mathrm{N}$ times and the position of its center in the object space is estimated after averaging over $\mathrm{N}$ position estimates as shown in Equation (3).

$$
\mathrm{P}_{\left(\phi_{\mathrm{m}}, \lambda_{\mathrm{m}}\right)}^{\mathrm{k}}=\sum_{i=1}^{\mathrm{N}} \frac{\mathrm{P}_{(\phi, \lambda)_{i}}^{\mathrm{k}}}{\mathrm{N}}
$$

$\mathrm{P}_{\left(\phi_{m}, \lambda_{m}\right)}^{k}$ represents the center of the k-th panel, which shall be used to generate a unique key to exploit the position information in order to access the DB.

\subsection{Subsystem Error Sources}

The proposed system involves various units within each subsystem such that each of the units introduces a certain amount of error that contributes to the total subsystem error budget. Currently the following error sources contributing are identified:

1. Payload altimeter resolution: A barometric altimeter is used to assess the UAV height $\mathrm{h}$ with a resolution of about $14 \mathrm{~cm}$. Such an error impacts $O^{\prime}$ i.e. image reference system determination.

2. UAV velocity: The UAV Ground Speed of typically less than $3 \mathrm{~m} / \mathrm{s}$ is taken into account with respect to the GNSS receiver position update.

3. Gimbal: The orientation of gimbal must be normal to object reference system, however, wind gusts may introduce for few milliseconds misalignment to be evaluated by tests. Typical resolutions for COTS gimbal is about $0.05^{\circ}$.

4. PV module Height: The height of PV panels is assessed in case of lack of information about PV panel placement in planar surface. This error source causes $\mathrm{P}_{\left(x, y, z_{0}=0\right)}$ not belonging to the object space.

5. PV module inclination: Panels have inclination which is typically a function of the latitude for PV plants on the ground. Such inclination reduces the cross section of the Panels as seen from UAV. 
6. Sensor lens distortion: Distortion introduced by sensors optics, to be assessed and mitigated during tests. Sense lens distortion account one of the most significant source of error budget.

7. GNSS accuracy: Error in position solution obtain from on-board RTK GNSS rover receiver contributes to the error budget. Further tests need to be conducted to quantify GNSS accuracy impact.

8. Sensor resolution: The error introduced by the resolution of commercial thermal cameras of available resolution up to $640 \times 480$ pixels.

9. Algorithm quantization: The errors introduced by the algorithm in the image reference system taking into account the panels dimensions expressed in pixels.

A thorough assessment of the aforementioned error sources shall be done during the test and validation phase of the study in order to quantify the contribution of the error sources to the subsystem error budget.

\section{GNSS Accuracy Requirement for UAV Positioning}

Considering the standard dimension of the solar panel the maximum error tolerance is $\min (50 \mathrm{~cm}, 80 \mathrm{~cm})$ to uniquely identify each solar panel regardless the inclination of the solar panel as shown in Figure 4 that. This implies that the subsystem error budget always be less than the maximum error tolerance

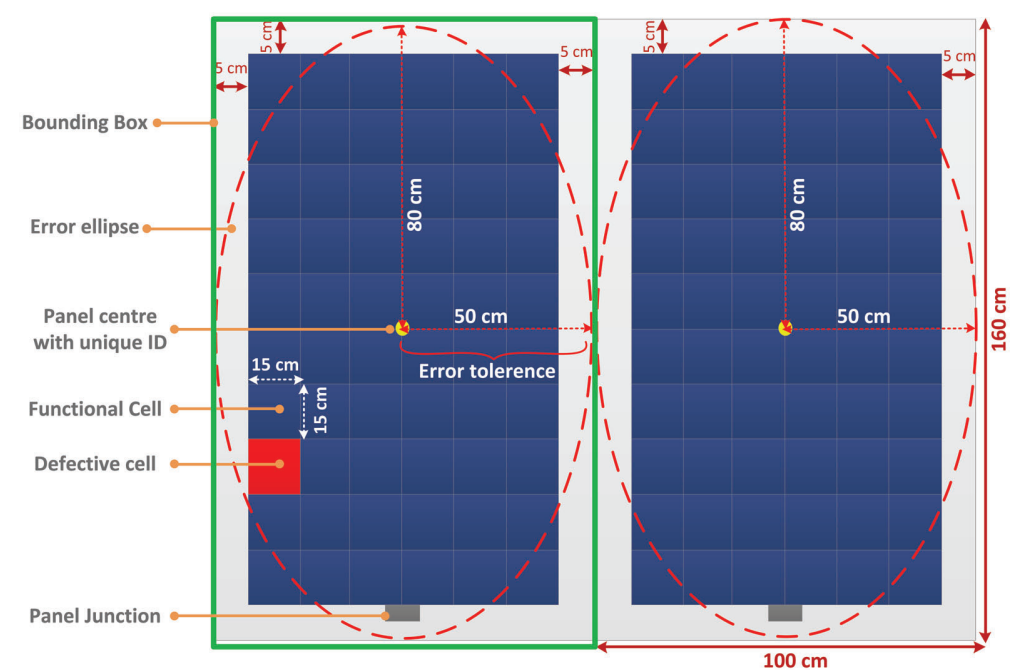

Figure 4 Standard solar panel dimensions, error ellipse, and maximum error tolerance. 
of $50 \mathrm{~cm}$. To meet subsystem error requirement, the accuracy of L1 RTK GNSS receiver is of paramount importance in order to restrict the GNSS positioning error contribution as minimum as possible keeping in view the low-cost, low-power and small form factor deployment constraints. Though, at this point, the exact positioning requirement is not known and shall be estimated during a dedicated validation and testing campaign. However, the expected GNSS positioning accuracy should be not more than $20 \mathrm{~cm}$ (2DRMS) to provide enough error margins for other error sources contributing to the subsystem error budget. It has to be remarked that the positioning accuracy requirement is subject to horizontal positioning only since the computer vision algorithms operate in two-dimensional space; therefore, no vertical positioning information is required.

Currently, a number of L1 RTK OEM receivers are available in market, which offer centimeter-positioning accuracy employing RTK messages in RTCM format. As listed in Table 1, ublox M8P [9] offers the least expensive L1 RTK solution with an added feature of integrated UHF radios for RTK RTCM message transmission/reception. The RTK RTCM messages can either be generated employing one of the OEM module as base station or it can be obtained from a public Continuously Operating Reference Station (CORS) such as EUREF permanent GNSS network [10] using Network Transport of RTCM via Internet Protocol (NTRIP) [11]. Typically, CORS is a desirable choice for using RTK in order to avoid base station setup complexity, however, the availability of CORS nearby PV plant is not always possible. For this reason, a local base station approach, which is supported by all the existing L1 RTK OEM receiver, presents a handy choice at the cost of base station installation setup for on-field operations in a PV plant.

Table 1 Market ready L1 GNSS RTK OEM Evaluation Boards

\begin{tabular}{lccc}
\hline Product Name & GNSS & Features & Est. Cost (€) \\
\hline ublox M8P & GPS/GLONASS & $\begin{array}{c}\text { Base-Rover/CORS, } \\
\text { UHF \& GNSS } \\
\text { Antenna }\end{array}$ & 340 \\
\hline Piksi & GPS & $\begin{array}{l}\text { Base-Rover, UHF } \\
\text { \& GNSS Antenna }\end{array}$ & 1000 \\
\hline Navspark S2525F8-BD-RTK & GPS/BeiDou & Base-Rover/CORS & 450 \\
\hline NV08C-EVK-RTK & GPS/GLONASS & Base-Rover/CORS & 1600 \\
\hline Reach RTK & GPS/GLONASS & Base-Rover/CORS & 500 \\
\hline
\end{tabular}




\section{Preliminary Results}

\section{1 ublox M8P Horizontal Positioning Accuracy}

ublox M8P base and rover equipped with active GPS/GLONASS Tallysman antenna, as shown in Figure 5, were installed on the rooftop of Aalborg University buildings at a spatial separation of 160 meter between base and rover as shown in Figure 6. The rover position data in NMEA format was logged at rate of $1 \mathrm{~Hz}$ for a period of three days. The logged file is then processed by RTKPLOT utility [12] to obtain rover ground track and related positioning statistics. Figure 7 shows plot of rover ground track considering

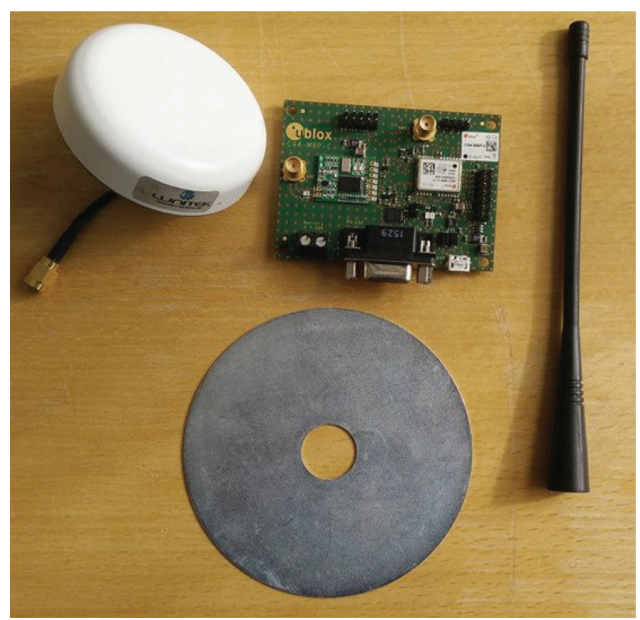

Figure 5 ublox M8P, Tallysman TW3400 antenna, ground plane, and 433 MHz UHF antenna for RTCM transmission/reception.
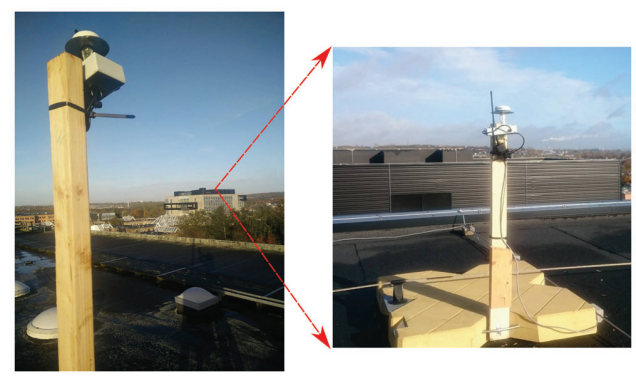

Figure 6 ublox M8P base (left) and rover (right) mounted on rooftop of Aalborg University buildings. The spatial separation between the rooftop of base and rover is $160 \mathrm{~m}$. 


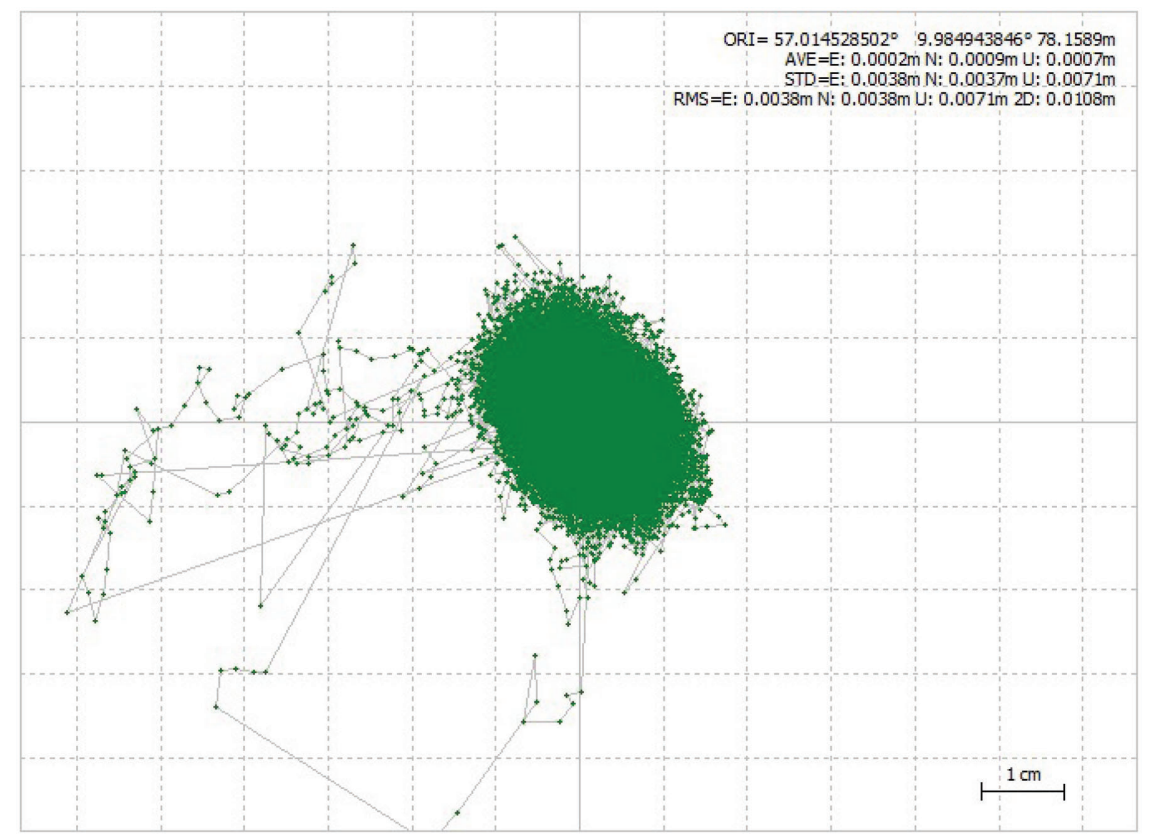

Figure 7 ublox M8P rover (static) horizontal positioning accuracy, 2DRMS $=1.08 \mathrm{~cm}$, and RTK Fix availability of $99 \%$.

position solution only with RTK fix. It can be observed that the rover provides 2DRMS positioning accuracy of $1.08 \mathrm{~cm}$, while the RTK fix was available 99\% of the time. Though the rover receiver was static, however, such positioning performance is significant for L1 RTK receiver considering the fact that no surveyed position was provided to the base receiver. Nevertheless, we expect that the degradation in positioning accuracy is inevitable under dynamic condition of moving UAV. We expect that positioning error should not exceed $20 \mathrm{~cm}$ in order to provide reasonable margin for other error sources. Unlike base, rover receiver benefits from improved signal reception as the GNSS signal experiences relatively less multipath and obstruction due to UAV flight at a height.

\subsection{Solar Panel Dimension Estimation from UAV}

A preliminary test was conducted in Castel Campagnano, Italy to estimate the dimensions of solar panel as a first of the proof-of-concept. A PV panel of known size $(68 \mathrm{~cm} \times 20.5 \mathrm{~cm})$ has been placed at a distance of 10 meters 
away from a reference point (a manhole) as shown in Figure 8. A number of optical images have been taken at different UAV height, the UAV is DJI Matrice 100 with a Zenmuse X3 gimbal and optical camera with a FOV of $84^{\circ}$ configured to record images with a resolution of $4000 \times 3000$ pixels in 4:3 format with the sensor at Nadir as shown in Figure 9.

Each captured image is enriched with gimbal attitude metadata (roll, pitch, azimuth height) and GNSS position referred to the centre of the image. It has to be remarked that the position is obtained from the pre-installed ublox M8N receiver on-board UAV, which does not support RTK positioning.

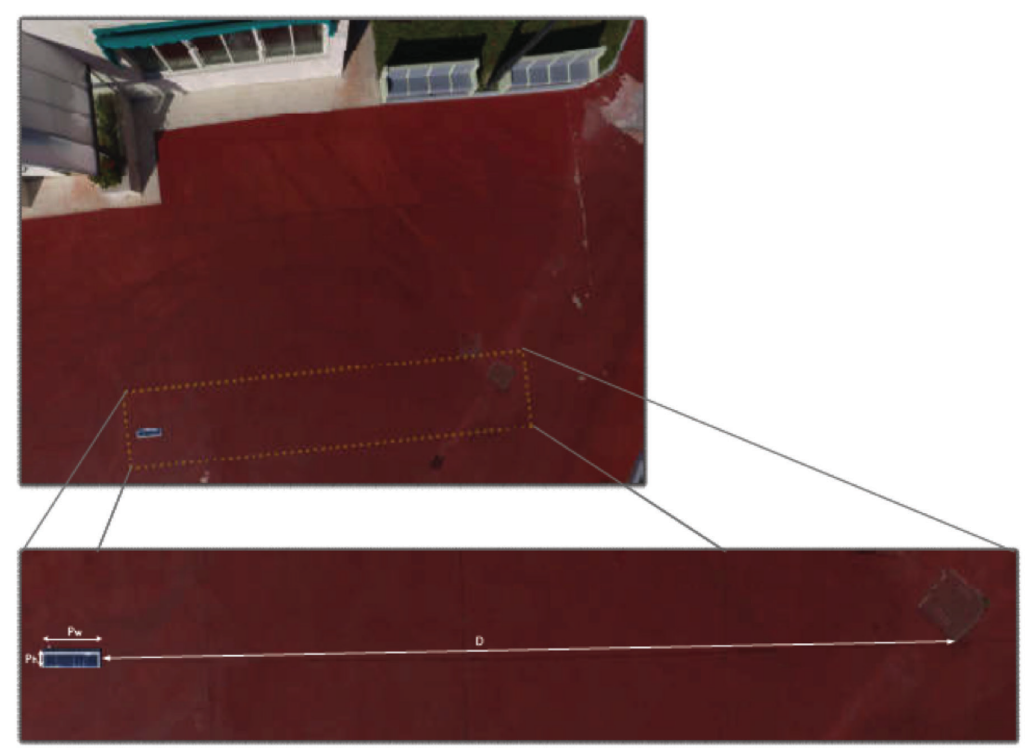

Figure 8 Testbed for the estimation of PV panel dimensions.

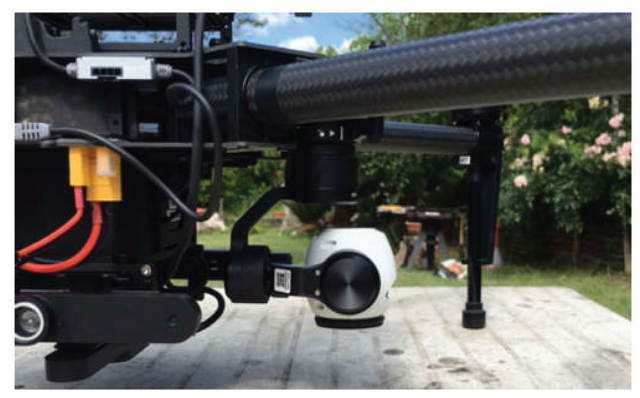

Figure 9 DJI MATRICE M100 with camera pointed at Nadir. 
The Ground Sampling Distance (GSD), which is the distance between two consecutive pixels measured on ground is calculated considering the image resolution expressed in pixel, the FOV of camera and the height of the UAV extracted from the EXIF/XMP (Exchangable Image Format/Extensible Metadata Platform) data embedded into images. The size of the panel expressed in pixels and the distance from the reference point has been detected in post processing and the equivalent dimensions in meter are calculated multiplying these values by the actual GSD. The height of Panel from the ground and its inclination that represents a contributing source of error is simplified by the exploitation of the ratio of the height and the width of the panel (reprsented by $\rho$ ) provided by the detected boundary of the image. To estimate the solar panel inclination, we first calculate the ratio $\rho$ then compare to the default ratio of the panel $\rho_{s}$. For example, the default aspect ratio for a $160 \mathrm{~cm} \times 100 \mathrm{~cm}$ PV panel is calculated as:

$$
\mathrm{GSD}=\frac{160}{100}=1.6
$$

If the ratio $\rho$ is less than the default ratio $\rho_{s}$ i.e. $\left(\rho<p_{s}\right)$ and the absolute value of their difference is greater than a threshold value $\left(\left(p-p_{s}\right)>T_{h}\right)$, then the panel is mounted with an inclination angle on the transversal side. In this case, the length of the longitudinal side $\mathrm{W}$ can be used as reference for calculating GSD as:

$$
\mathrm{GSD}=\frac{\mathrm{W}[\mathrm{m}]}{\mathrm{W}[\text { Pixel }]}
$$

On the other hand, if $\rho>\rho_{s}$ and $\left(p-p_{s}\right)>T_{h}$ then the panel is inclined on the longitudinal side and we use the length of the transversal side $\mathrm{H}$ as reference for calculating GSD as:

$$
\mathrm{GSD}=\frac{\mathrm{H}[\mathrm{m}]}{\mathrm{W}[\text { Pixel }]}
$$

Following this approach, images at different UAV heights were taken for which

\begin{tabular}{|c|c|c|c|c|c|c|c|}
\hline $\begin{array}{l}\text { UAV } \\
\text { Height [m] }\end{array}$ & $\begin{array}{c}\text { GSD } \\
{[\mathrm{m} / \mathrm{px}]}\end{array}$ & $\mathrm{W}[\mathrm{Px}]$ & $\mathrm{W}$ [m] & $\mathrm{H}[\mathrm{Px}]$ & $\mathrm{H}[\mathrm{m}]$ & $\begin{array}{l}\text { Ref. Point } \\
\text { Dist. }[\mathrm{Px}]\end{array}$ & $\begin{array}{l}\text { Ref Point } \\
\text { Dist. [m] }\end{array}$ \\
\hline 7.8 & 0.0029437 & 231 & 0.68000 & 69 & 0.2031169 & 3179 & 9.3580952 \\
\hline 12.6 & 0.0044444 & 153 & 0.68000 & 45 & 0.2000000 & 2230 & 9.9111111 \\
\hline 15.8 & 0.0074725 & 91 & 0.68000 & 27 & 0.2017582 & 1370 & 10.2373626 \\
\hline
\end{tabular}
the measurements are provided in Table 2. It can be observed that solar panel

Table 2 Estimated PV dimensions 
dimensions are precisely estimated at 12.5 meter UAV height, while at heights lower or greater than $12.5 \mathrm{~m}$, marginal errors in solar panel estimation can be noticed. These errors can be mitigated using narrower FOV and smaller sensor area. Though the approach showed promising results in estimating the solar panel dimension, however, using thermal camera that offers lower resolution than the visual camera it would be interesting to see how better this approach works.

\section{Conclusion}

The target market of the proposed solution comprises of both maintainers and owners of large PV plants as final users, which are encouraged to use customized UAV payload design together with UAV ground segment to enhance PV plant energy production in a cost-effective way by automating PV plant maintenance.

As a first step towards a market-ready solution aligned with PV maintenance user needs, this paper presented system architecture and subsystem design at a glance as well as expected error sources contributing to the total error budget. Furthermore, the paper discussed the end-to-end algorithm, which performs the key tasks of direct geo-referencing, image acquisition, panel recognition, faulty module detection, and solar panel cataloguing in database. Furthermore, low-cost L1 GNSS RTK positioning solution is briefly discussed along with the expected error contribution to the overall subsystem error budget. The paper presented static horizontal positioning accuracy of ublox M8P, which indicated that M8P is a potential candidate GNSS RTK receiver to be integrated on-board UAV. Finally, solar panel dimension estimation by means of DJI MATRICE-100 UAV equipped with visual sensor and a non-RTK GNSS receiver is presented. The preliminary tests were successful in establishing the proof-of-concept, however, further tests are required that considers a thermal camera instead of visual camera, ublox M8P GNSS RTK receiver, and a complete end-to-end algorithm evaluation. In the upcoming phase of the project, a thorough test and validation campaign shall be performed in order to realize the proposed concept and end-to-end algorithm taking in to account on-field PV plant conditions.

\section{Acknowledgment}

The work presented in this paper is a part of the ongoing activities performed in the framework of EGNSS high Accuracy SYstem for the maintenence 
of PhotoVoltaic plants (EASY-PV). The project is sanctioned under H2020Galileo-2015-1 call for Small and Medium Enterprise (SME) based EGNSS application having grant agreement number 687409 . The project started off on Feb. 01, 2016 and shall end on Jan. 31, 2018. The project is coordinated by Sistematica S.p.A with contributions from other partners, namely, BSS Aarhus University, TopView srl, DeepBlue, Entec, and Alpha consultants.

\section{References}

[1] Gary, B., and Adrian, K. (2008). Learning OpenCV: Computer vision with the OpenCV library. O'Reilly Media, Incorporated.

[2] Cai, G., Chen, B. M., and Lee, T. H. (2011). Unmanned Rotorcraft Systems. Springer Science \& Business Media.

[3] Vincenty, T. (1975). Direct and inverse solutions of geodesics on the ellipsoid with application of nested equations. Survey review, 23, 88-93.

[4] Karney, C. F. (2013). Algorithms for geodesics. J. Geodesy 87, 43-55.

[5] Kraus, K. (1998). Photogrammetry. Ferdinand Dummlers Verlag, vol. 1.

[6] Kraus, K. (1997). Photogrammetry. Ferdinand Dummlers Verlag, vol. 2.

[7] Cramer, M. (2001). Performance of GPS/inertial solutions in photogrammetry.

[8] Hough, P. V. (1962). Method and means for recognizing complex patterns (No. US 3069654).

[9] Mongrdien, C., Doyen, J.-P., Strom, M. and Ammann, D. (2016). "Centimeter-Level Positioning for UAVs and Other Mass-Market Applications," in Proceedings of the 29th International Technical Meeting of the Satellite Division of the Institute of Navigation (ION GNSS+), Portland, Oregon.

[10] Bruyninx, C. (2004). "The euref permanent network: a multi-disciplinary network serving surveyors as well as scientists," GeoInformatics 7, 32-35.

[11] Weber, G., Dettmering, D., and Gebhard, H. (2005). "Networked transport of rtcm via internet protocol (ntrip)," in A Window on the Future of Geodesy. Springer, 60-64.

[12] Takasu, T., Kubo, N., and Yasuda, A. (2007). "Development, Evaluation and Application of rtklib: a program library for rtk-gps," in GPS/GNSS symposium, 213-218. 


\section{Biographies}

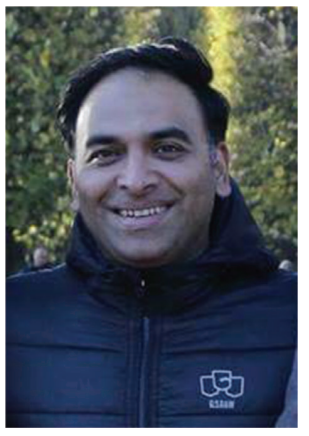

Bilal Muhammad holds a Ph.D in Telecommunication Engineering from the University of Rome Tor Vergata, Italy. He is working as a postdoc at the Department of Business Devleopment and Technology, Aarhus University. His research focus is on the use of GNSS in aviation and mass-market user applications. Currently, he is involved in EU H2020 projects focusing on the application of Remotely-Piloted Aircraft System in a multitude of businesses, which include, Inspection and Monitoring, Search and Rescue, and Precision Agriculture.

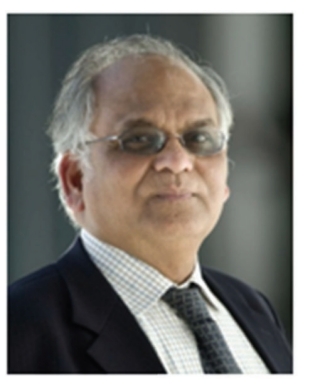

Ramjee Prasad, Fellow IEEE, IET, IETE, and WWRF, is a Professor of Future Technologies for Business Ecosystem Innovation (FT4BI) in the Department of Business Development and Technology, Aarhus University, Herning, Denmark. He is the Founder President of the CTIF Global Capsule (CGC). He is also the Founder Chairman of the Global ICT Standardisation Forum for India, established in 2009. GISFI has the purpose of increasing 
of the collaboration between European, Indian, Japanese, North-American and other worldwide standardization activities in the area of Information and Communication Technology (ICT) and related application areas.

He has been honored by the University of Rome "Tor Vergata", Italy as a Distinguished Professor of the Department of Clinical Sciences and Translational Medicine on March 15, 2016. He is Honorary Professor of University of Cape Town, South Africa, and University of KwaZulu-Natal, South Africa.

He has received Ridderkorset af Dannebrogordenen (Knight of the Dannebrog) in 2010 from the Danish Queen for the internationalization of top-class telecommunication research and education.

He has received several international awards such as: IEEE Communications Society Wireless Communications Technical Committee Recognition Award in 2003 for making contribution in the field of "Personal, Wireless and Mobile Systems and Networks", Telenor's Research Award in 2005 for impressive merits, both academic and organizational within the field of wireless and personal communication, 2014 IEEE AESS Outstanding Organizational Leadership Award for: "Organizational Leadership in developing and globalizing the CTIF (Center for TeleInFrastruktur) Research Network", and so on.

He has been Project Coordinator of several EC projects namely, MAGNET, MAGNET Beyond, eWALL and so on.

He has published more than 40 books, 1000 plus journal and conference publications, more than 15 patents, over $100 \mathrm{PhD}$ Graduates and larger number of Masters (over 250).

Under his leadership, magnitudes of close collaborations are being established among premier universities across the globe. The collaborations are regulated by guidelines of the Memorandum of Understanding (MoU) between the collaborating universities. 


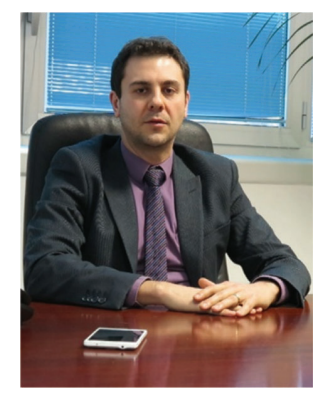

Marco Nisi has a master degree in "Aerospace Engineering" attained in "La Sapienza, Rome" and a post-graduate master's degree with highest honours in "Advanced Navigation and Communication Satellite Systems" achieved in "Tor Vergata, Rome".

He's a project manager, system engineer and SW engineer experienced (10 years) at working in complex national and international environments. He managed several projects exploiting GNSS as a Key Enabling Technology for both mass market and professional applications with main focus in Energy, Security and NAVCOM aeronautics domains.

He is author of numerous articles on the topic of RPAS and co-author of GREAT (GNSS Regenerative Encryption Algorithm and technique) solution which has been awarded by FINMECCANICA as "2009 Innovation Award" competition winner.

He's also performing System Design and Risk Analysis activities related to Galileo System and PRS User Segment frameworks as a consultant.

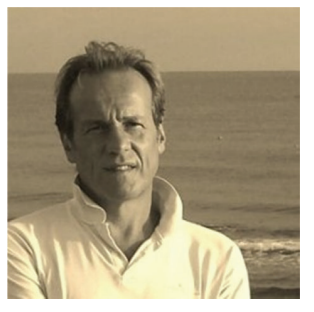

Fabio Menichetti has a master degree in "Electronic Engineering" attained in "La Sapienza, Rome".

He started in international companies where has acquired solid experiences as System Engineer, Project Manager, Product Manager. 
He's a Program Manager, system engineer and SW engineer experienced (more than 25 years) at working in complex national and international environments. He managed several projects in Aeropsace program as Cosmo Sky Med and Satellite Communications.

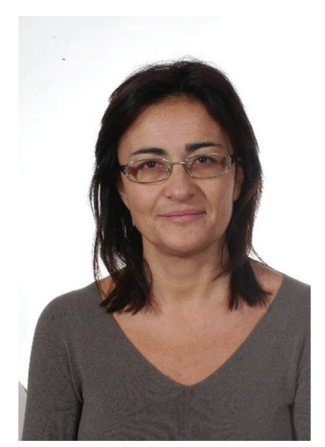

Ernestina Cianca is Assistant Professor at the Dept. of Electronic Engineering of the University of Rome Tor Vergata, where she teaches Digital Communications and ICT Infrastructure and Applications (WSN, Smart Grid, ITS etc.). She is the Director of the II Level Master in Engineering and International Space Law in Satellite systems for Communication, Navigation and Sensing. She is vice-director of the interdepartmental Center CTIF-Italy. She is the Editor-in Chiefs of the CONASENSE (Comm/Nav/Sensing and services) Journal, River Publishers.

She has worked on wireless access technologies (CDMA, OFDM) and in particular in the waveforms design, optimization and performance analysis of radio interfaces both for terrestrial and satellite communications. An important part of her research has focused on the use of EHF bands (Q/V band, W band) for satellite communications and on the integration of satellite/terrestrial/HAP (High altitude Platforms) systems. Currently her main research interests are in the use of radio-frequency signals (opportunistic signals such as WiFi or specifically designed signals) for sensing purposes, and in particular. Device-free RF-based activity recognition/crowd counting/density estimation and localization; UWB radar imaging (i.e., stroke detection). She is author/ co-author of 100 papers in international journals and conferences. 


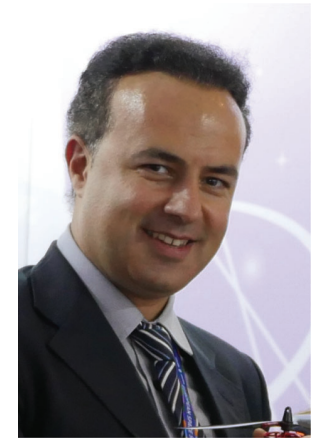

Alberto Mennella holds a B.Sc degree Telecommunication Engineering and M.Sc Degree in Nautical Sciences with a specialization in Radio Navigation, achieved in 2004 at "University of Naples Parthenope", Italy. He worked in various companies in the aerospace industry such as Thales Alenia Space Italy in different space programs and in companies operating in industrial research and software development. In December 2009, he was awarded with the "Thales Innovation Awards" for a "GEO Encryption" algorithm. In April 2013 he had designed and built a validation platform for a NAVCOM algorithm based on a multi-rotor system (RPAS) capable of autonomous flight (GNSS, SBAS), aimed to test and validate a prototype of user terminal which led him to fund TOPVIEW SRL, an innovative start-up company focused on high added value services for the emerging drone market. From October 2017 he participates in SESAR U-space program for the definition of unmanned drone traffic in Europe. At present time he coordinates main R\&D projects in TOPVIEW SRL, with particular reference to H2020 programs.

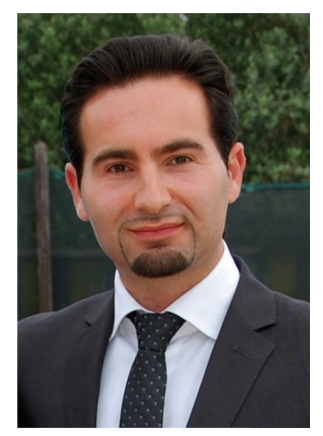

Graziano Gagliarde holds a B. Sc. degree in Telecommunication Engineering from University of Sannio, Italy. He collaborates with Topview since April 
2015 and he currently works as a software designer. He developed the software for a hybrid optical/thermal sensor (based on the Flir Lepton module) used in search and rescue operations by Coast Guard to detect the heat generated by survivor's head in the sea. Within various research projects he has acquired a solid experience on collision detection and tracking computer vision algorithms applied to multi-core architecture and parallel programming models. The variety of sensors (camera, laser rangefinder, IMUs) available onboard the UAV has improved considerably his knowledge of the communication protocols and standards used in most popular flight management units. He has been also involved in $\mathrm{H} 2020$ european project Easy-PV for the design and development of a software platform able to identify and georeference, trough computer vision and geodesics algorithms, the defective PV modules in large photovoltaic plants.

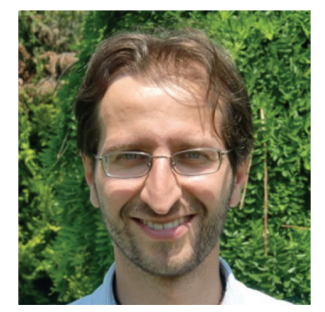

Davide Marenchino is a civil engineering, graduated at the Politecnico of Turin in 2004. He attained a Ph.D. in "Land and Environment" in Turin, working on researches in the geomatic field. In particular, he developed automatic procedures for the automatic extraction of $3 \mathrm{D}$ data from images acquired by UAV (Unmanned Aerial Vehicle). He has publisched more than 20 papers in national and international periodicals.

He has worked at Entec spa, a company which works in the renewable energy field, since 2010. Nowadays, he is a technical engineering in Entec, with tasks concerning design and management of hydropower, windpower and photovoltaic plants. The main activities concern hydrological and wind assessments, land analysis with GIS techniques and thermal analysis on photovoltaic modules. 
